\title{
Open Source PhotoVoltaics Library for Systemic Investigations
}

\author{
Jovan Brkic ${ }^{1} \quad$ Muaz Ceran $^{1} \quad$ Mohamed Elmoghazy $^{1} \quad$ Ramazan Kavlak $^{1}$ \\ Anton Haumer ${ }^{2} \quad$ Christian Kral $^{1}$ \\ ${ }^{1}$ TGM Wien XX, College of Engineering, Austria, dr.christian.kral@gmail.com \\ ${ }^{2}$ OTH Regensburg, Germany, anton. haumer@oth-regensburg.de
}

\begin{abstract}
For the planning of photovoltaic power plants standard software tools are used. Most of these software tools use statistical solar data to determine the overall energy harvest of a photovoltaic plant over one year. The calculations rely on stationary location and ideal boundary conditions, e.g., constant ambient temperature. Even though, for example, shadowing may be considered by standard software, the investigation of untypical configurations and problems cannot be performed by such software, as most configurations cannot be changed by the user.

The presented PhotoVoltaics library was developed with the intention to provide a flexible framework for standard and non-standard problems. Particularly, the PhotoVoltaics library can be coupled with other Modelica libraries to perform systemic investigations. An application library, PhotoVoltaics_TGM, is provided as add-on, where measured data of two photovoltaic pants of the TGM in Vienna can be compared with simulation results. This add-on library serves as validation of the PhotoVoltaics library.
\end{abstract}

Keywords: Photovoltaics, cell, module, plant, data sheet, converter, maximum power tracking, irradiance, terrestrial solar model

\section{Introduction}

For academic and scientific investigations and calculations in the engineering field of photovoltaics an open source library is advantageous. Currently, some Modelica libraries exist which provide photovoltaic plans on different levels of abstractions.

The Buildings library (Wetter, 2017) includes photovoltaic plant models based area of cross section and efficiency parameters. The plant models evaluate the irradiance input and calculate the harvested power by means of a non-standard electrical connector. Additionally, the Buildings library provides blocks for the processing of public irradiance data available from EnergyPlus.
In the BuildSysPro library a photovoltaic model is provided based on physical material and geometry parameters, but not electrical parameters (BuildSysPro, 2017). Electric power is represented by a signal output, so the actual interaction with maximum power control and the power grid cannot be modeled in a physical way.

The PVSystems library relies on manufacturer data and uses electrical connectors from the Modelica Standard library (PVSystems, 2017). The photovoltaics model includes a series and parallel resistor but unfortunately no parameterization aid is provided to determine the resistance parameters. Temperature dependency is considered by means of a temperature signal input. Even though the PVSystems library relies on a roughly similar physical modeling approach as the PhotoVoltaics library, the model behavior is not consistent. The investigation of the open circuit voltage at standard conditions and a slightly higher temperature shows simulations results which clearly deviate from the data sheet parameters.

The PhotoVoltaics library was developed during a Diploma project at the College of Engineering, TGM, in 2016--17. It is available on GitHub (Kral, 2017). The main target of this library was to provide physical models of photovoltaic components that show consistent behavior and can be parameterized solely on data sheet values. So the open circuit voltage and short circuit current including their temperature dependencies were intended to be modeled to fully match the given data sheet values.

The paper presents the structure of the provided libraries in Section 2. In Section 3 the data sheet parameters are presented and explained. Based on these parameters, the included cell, module and plant model are elaborated in Section 4. Different converters and the maximum power tracking are explained in Sections 5 and 6. The irradiance models of Section 7 are needed for the system investigations of complex photovoltaic systems. The library validation and further application examples are presented in Sections 8 and 9 . 


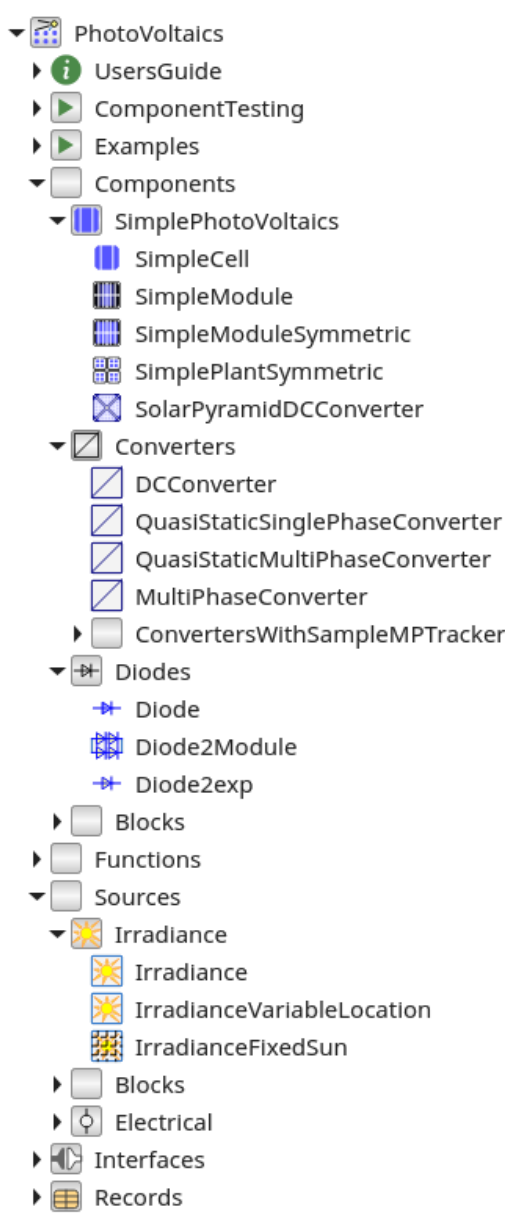

Figure 1. Structure of the PhotoVoltaics library

\section{Library Structure}

The PhotoVoltaics library includes:

- Photovoltaic (PV) components (cells, modules and plants)

- Converters (DC/DC, quasi static single and three phase, transient three phase)

- Diodes

- Analytic irradiance models (terrestrial, arbitrary sun location)

- Records of selected industrial module data sheets

The structure of the library is depicted in Figure 1. General configuration examples are included in the package Examples. Additional application examples which allow the validation of the proposed models are provided in the external package PhotoVoltaics_TGM shown in Figure 2.

\section{Data Sheet Parameters}

Most data sheet parameters refer to standard conditions (STC) of a module. These conditions are characterized by

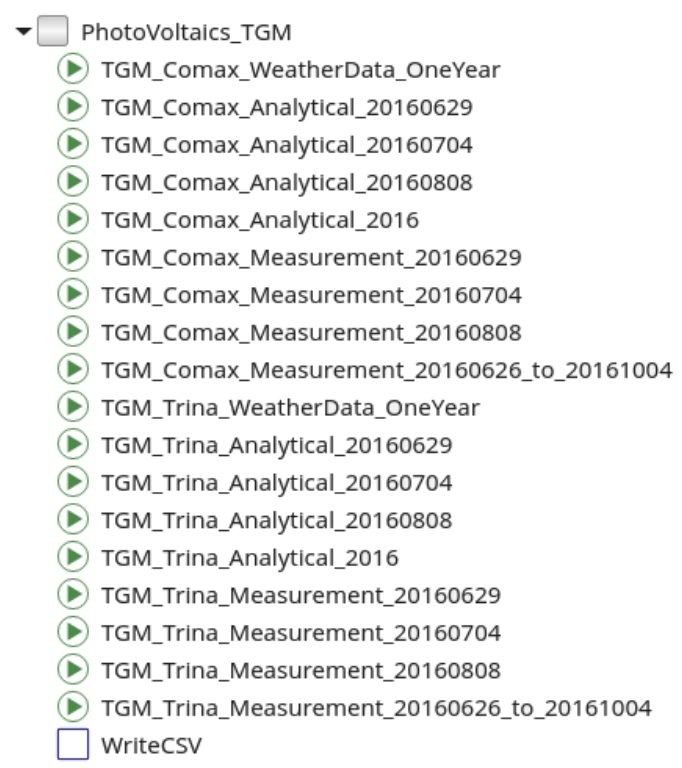

Figure 2. Structure of the PhotoVoltaics_TGM library, including application and validation examples

the reference temperature $T_{\text {ref }}=25^{\circ} \mathrm{C}$ and the reference irradiance $_{\text {irradiance }}$ ref $=1000 \mathrm{~W} / \mathrm{m}^{2}$. Under these reference conditions the following quantities are listed:

- Open circuit voltage $V_{\text {oc,ref }}$ under reference conditions

- Short circuit current $I_{\mathrm{sc}, \text { ref }}$ under reference conditions

- Maximum power voltage $V_{\mathrm{mp} \text {,ref }}$ under reference conditions

- Maximum power current $I_{\mathrm{mp}, \text { ref }}$ under reference conditions

- Linear temperature coefficient of open circuit voltage $\alpha_{V \text { oc,ref }}$ at reference conditions

- Linear temperature coefficient of short circuit current $\alpha_{\text {Isc,ref }}$ at reference conditions

Typically, the temperature coefficient of maximum power is also listed in the data sheet of a photovoltaic module. This parameter is, however, not evaluated in the proposed model, since the model inherently considers the maximum power temperature coefficient from the open and short circuit temperature coefficients.

Each module is usually equipped with nb bypass diodes. These diodes are usually connected anti parallel to two or more strings of the photovoltaic cells. The diodes are used to overcome reverse operating conditions caused by partial shading of the module. In order to consider the bypass diodes properly in the model, the parameters BvCell, Ibv and Nbv listed in Listing 1 are taken into account.

Photovoltaic modules usually consist of ns series connected cells, but have no parallel connected cells. The cell parameters are determined from the module parameters as 
shown in Listing. 1. Additionally, the temperature voltage at reference temperature,

$$
V_{t, \mathrm{ref}}=\frac{k \cdot T_{\mathrm{ref}}}{Q}
$$

is calculated as final parameter. In this equation $k$ is the Boltzmann constant and $Q$ is the elementary charge (of an electron). The module parameters are organized as record in Modelica.

Listing 1. Record of module data

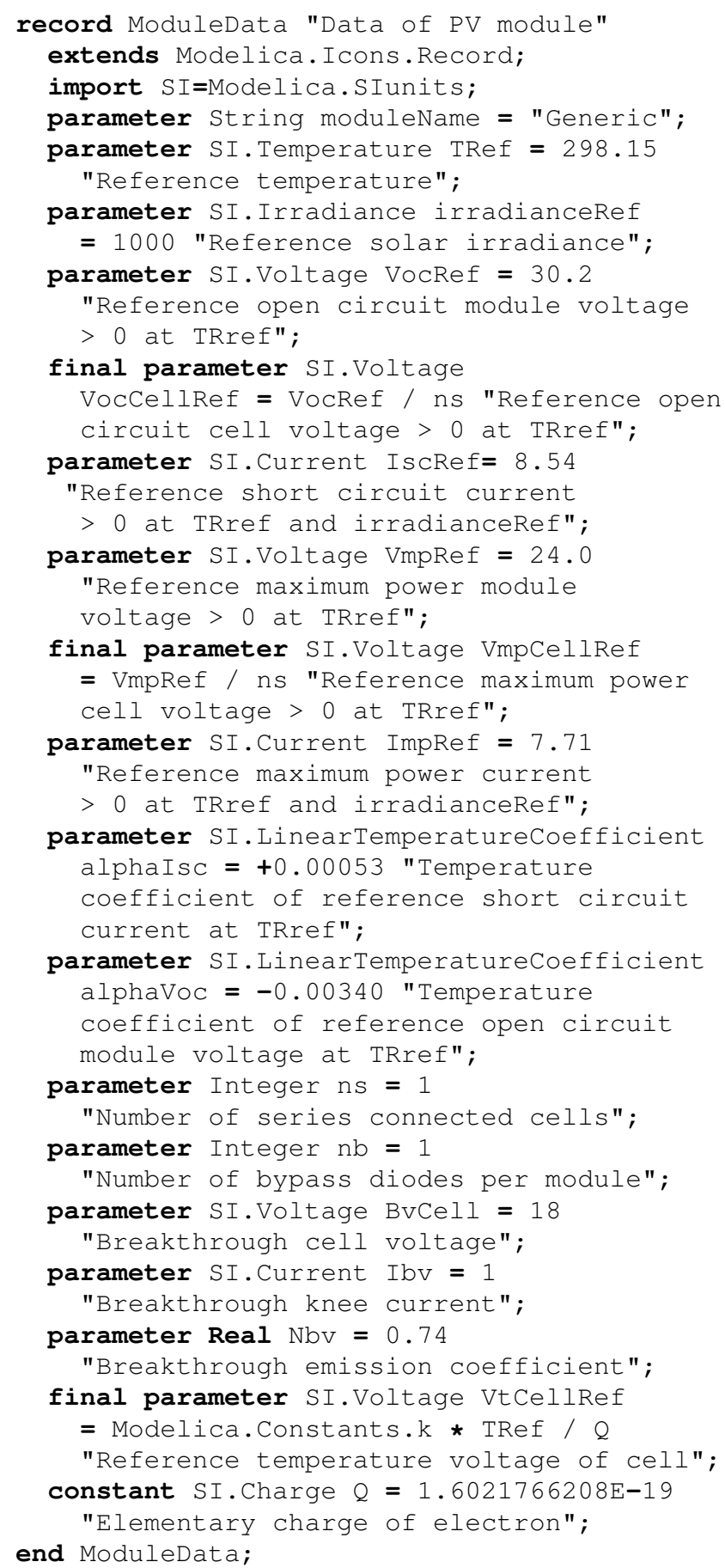

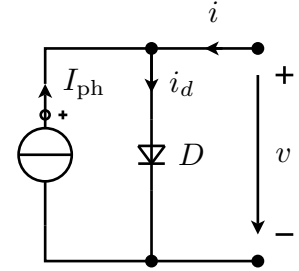

(a)

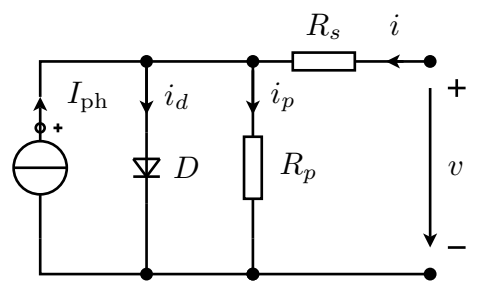

(b)
Figure 3. (a) Basic and (b) extended equivalent circuit diagram of PV cell

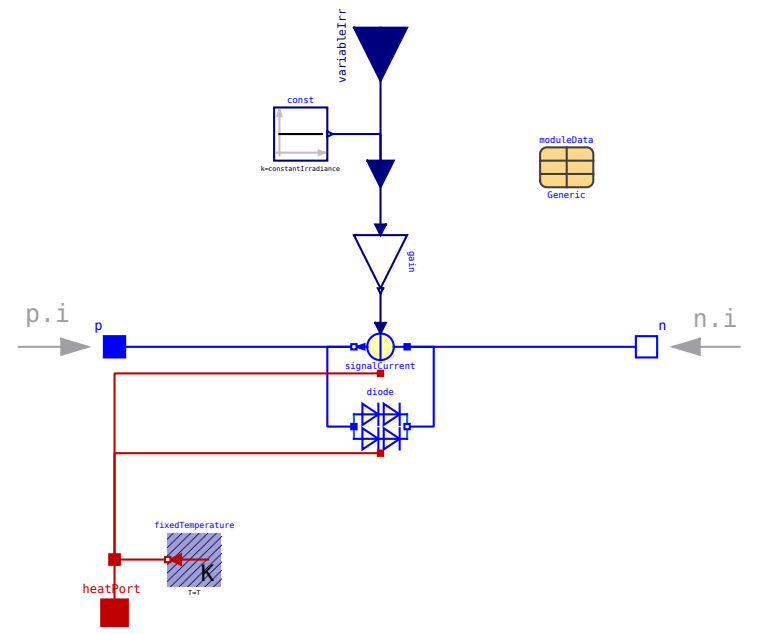

Figure 4. Modelica implementation of the cell model SimpleCell

\section{PhotoVoltaics Components}

\subsection{Photo Voltaic Cells}

The basic photovoltaic component is the cell. The implemented model consists of a diode and current source as shown in Figure 3 (Mahmoud et al., 2012). The current source represents the solar power source and the diode includes the semiconductor properties of a cell. The basic equivalent circuit diagram of Figure 3(a) could be extended by a series resistor $R_{s}$ and a parallel resistor $R_{p}$. The extended model may be more accurate than the basic model, but from data sheet values the extended model can usually not be parameterized as the slopes of the voltage versus current characteristics are not provided accurately enough by the manufacturers. Therefore, the PhotoVoltaics library includes only basic models, so far. The actual implementation of the cell model SimpleCell is depicted in Figure 4.

In the basic photovoltaic cell model the source current $I_{\mathrm{ph}}$ is modeled directly proportional to the actual irradiance the cell is exposed to, including temperature dependence

$$
\frac{I_{\mathrm{ph}}}{I_{\mathrm{ph}, \mathrm{ref}}}=\frac{\text { irradiance }_{\text {irradiance }}}{\text { iref }}+\alpha_{I \mathrm{sc}}\left(T-T_{\mathrm{ref}}\right),
$$

where $T$ is the actual operating temperature of the current source. The actual irradiance can be considered in two 


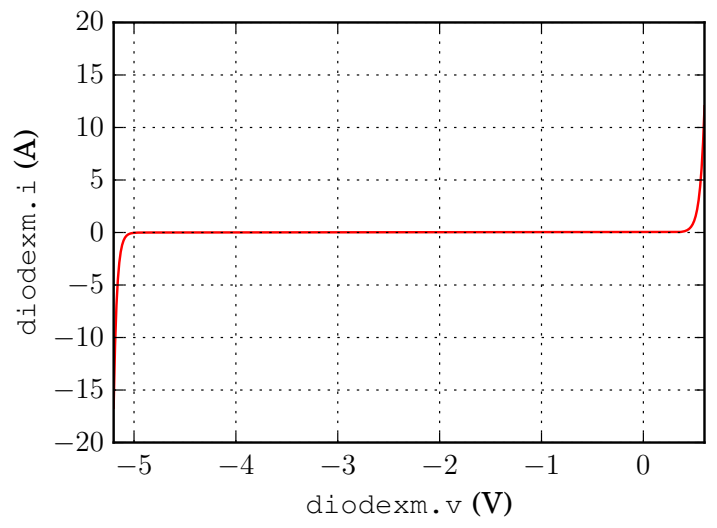

Figure 5. Diode model with two exponential regions and one linear region

ways:

- A constant quantity constantIrradiance is provided as parameter if the boolean parameter useconstantIrradiance = true

- A signal input variableIrradiance is enabled if the boolean parameter useConstantIrradiance = false

The variable irradiance in (2) is thus assigned to either constantIrradiance or variableIrradiance depending on the boolean parameter useConstant Irradiance.

The diode models is especially designed for the purpose of photovoltaic applications. It covers the forwards and backwards breakthrough region. For numerical reasons the current versus voltage characteristic of the used diode model considers three different regions:

- Forward exponential range for positive cell voltages,

$$
i=I_{d s}\left(\exp \left(\frac{v}{m V_{t}}\right)-1\right)+\frac{v}{R},
$$

where $R=10^{8} \Omega$ is a parallel resistance used to stabilize the model numerically; $I_{d s}$ represents the saturation current $m$ is the ideality factor of the diode

- Backward linear range in the reverse direction starting from zero voltage

- Backward exponential range of negative voltages in the breakthrough region

The current versus voltage characteristic of the implemented diode model depicted in Figure 5 is determined by the experiment DiodeCompare using $\mathrm{ns}=1$, nsmodule $=1$ and npModule $=1$. The variables $I_{d s}$ and $m$ of the diode forward exponential region (3) are determined through the operating conditions of the photovoltaic cell. The first condition is derived from the open circuit case of Figure 3(a) substituted in (3),

$$
I_{\mathrm{sc}}=I_{d s}\left(\exp \left(\frac{V_{\mathrm{oc}}}{m V_{t}}\right)-1\right) \text {. }
$$

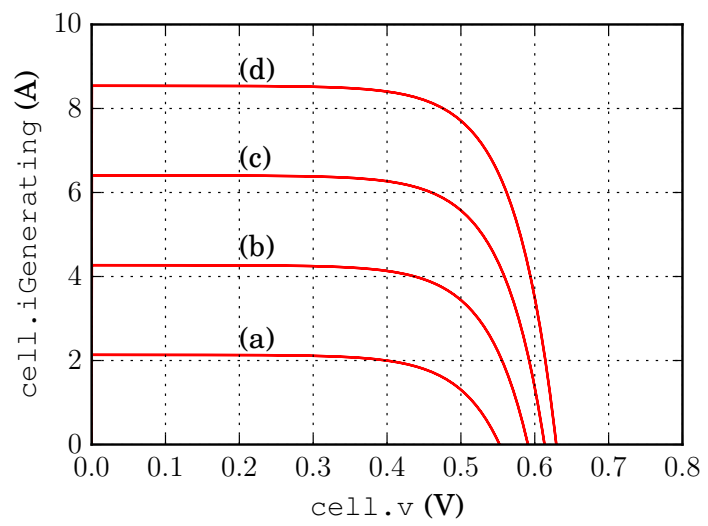

Figure 6. Cell current versus cell voltage for (a) irradiance $=$ $250 \mathrm{~W} / \mathrm{m}^{2}$, (b) irradiance $=500 \mathrm{~W} / \mathrm{m}^{2}$, (c) irradiance $=$ $750 \mathrm{~W} / \mathrm{m}^{2}$, and (d) irradiance $=1000 \mathrm{~W} / \mathrm{m}^{2}$

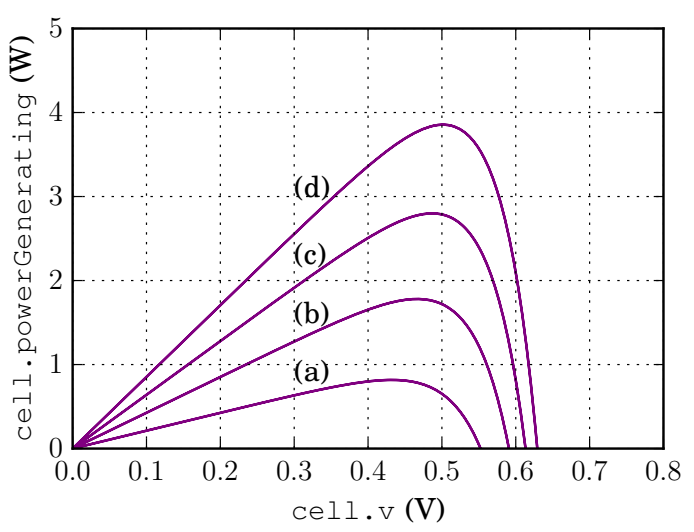

Figure 7. Cell power versus cell voltage for (a) irradiance $=$ $250 \mathrm{~W} / \mathrm{m}^{2}$, (b) irradiance $=500 \mathrm{~W} / \mathrm{m}^{2}$, (c) irradiance $=$ $750 \mathrm{~W} / \mathrm{m}^{2}$, and (d) irradiance $=1000 \mathrm{~W} / \mathrm{m}^{2}$

In this equation the photo current is equal to the short circuit current. The impact of the parallel resistance on this equation is neglected. The second conditions is determined by the temperature voltage

$$
V_{t}=\frac{k \cdot T}{Q} .
$$

Additionally the temperature dependencies of the open circuit and short circuit voltage of the photovoltaic cell have to be taken into account:

$$
\begin{gathered}
V_{\mathrm{oc}}=V_{\mathrm{oc}, \text { ref }}\left(1+\alpha_{V \mathrm{oc}}\left(T-T_{\text {ref }}\right)\right) \\
I_{\mathrm{sc}}=I_{\mathrm{sc}, \text { ref }}\left(1+\alpha_{I \mathrm{oc}}\left(T-T_{\text {ref }}\right)\right)
\end{gathered}
$$

This implementation causes the saturation current $I_{d s}$ and the ideality factor $m$ to be temperature dependent. The variability of these quantities is a result of consistent operating conditions of the proposed model based on manufacturer data.

The linear scaling of the short circuit current according to (2) for constant temperature is demonstrated in Figure 6. Figure 7 shows the dependence of the maximum 


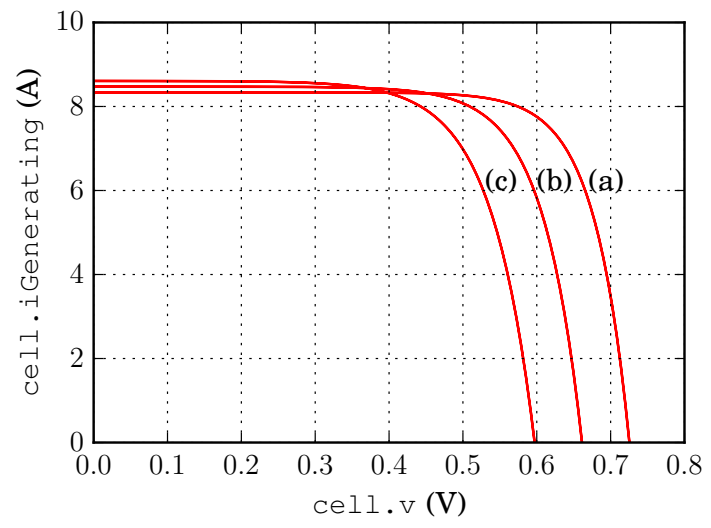

Figure 8. Cell current versus cell voltage for (a) $T=-20^{\circ} \mathrm{C}$, (b) $\mathrm{T}=10^{\circ} \mathrm{C}$, (c) $\mathrm{T}=40{ }^{\circ} \mathrm{C}$

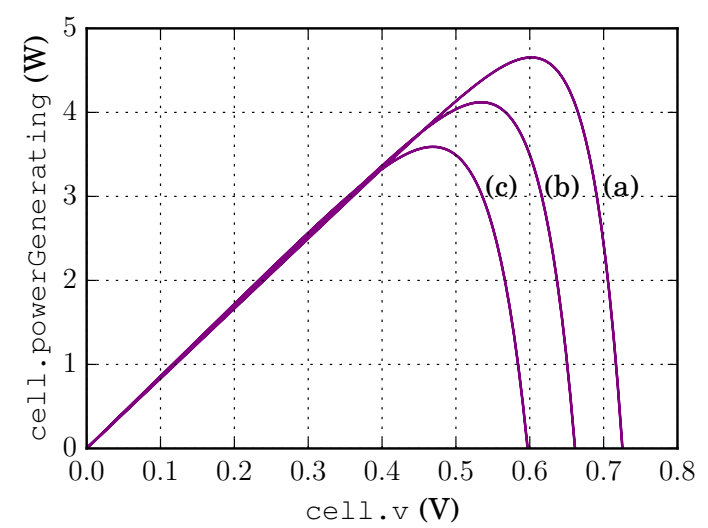

Figure 9. Cell power versus cell voltage for (a) $\mathrm{T}=-20^{\circ} \mathrm{C}$, (b) $\mathrm{T}=10^{\circ} \mathrm{C}$, (c) $\mathrm{T}=40^{\circ} \mathrm{C}$

power point on irradiance. The impact of the operating temperature on the short circuit current and the open circuit voltage is shown in Figure 8. In this figure a typical case is evaluated, considering a positive temperature coefficient of the short circuit current and a negative temperature coefficient of the open circuit voltage. Since the absolute value of the temperature coefficient of the open circuit is greater than the temperature coefficient of the short circuit current, the temperature coefficient of the maximum power is negative. Consequently, the maximum power harvest of a photovoltaic cell decreases with increasing temperature, see Figure 9.

The photovoltaic cell model also considers shading of a cell. In this implementation shadow $=0$ represents the case of full exposure to solar irradiance, whereas shadow $=1$ considers zero irradiance. In addition to conventional shading caused by visible obstacles, this approach also allows the consideration of the dimming of the cell over time due the impact of pollution (Häberlin and Renken, 1999; Renken and Häberlin, 1999).

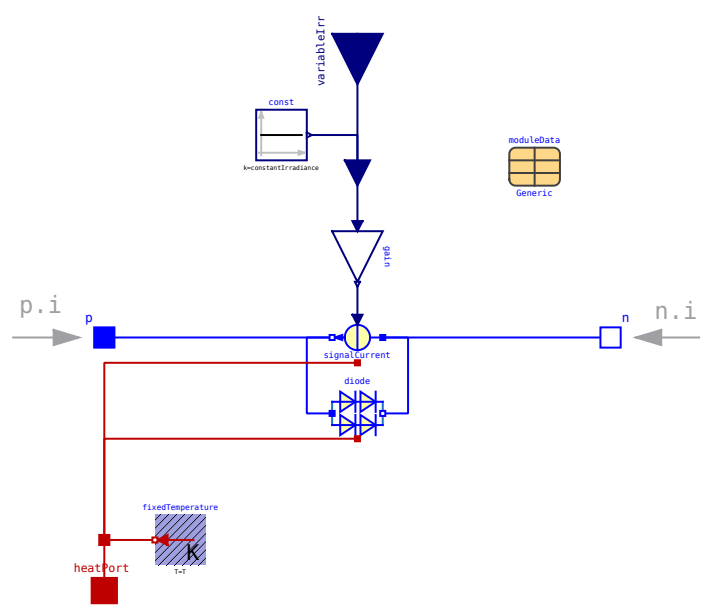

Figure 10. Modelica implementation of the symmetric module model SimpleModulesymmetric

\subsection{Symmetric Photovoltaic Modules}

In the PhotoVoltaics library two different module classes are provided. The symmetric module assumes uniform shading of all cells of a module. In this case the bypass diodes are not taken into account.

The cell currents $i \mathrm{Cell}$ and the cell voltages vCell correspond with the module current $i$ and the module voltage $\mathrm{v}$ by

$$
\begin{aligned}
\mathrm{iCell} & =\mathrm{i}, \\
\mathrm{ns} * \mathrm{vCell} & =\mathrm{v} .
\end{aligned}
$$

In (8) it is considered that a module has no parallel connections. However, both the current source and the diode model are designed such way that parallel and series connections may be considered by scaling the photo current and the diode voltage and current, respectively. Therefore, the SimpleModulesymmetric model of Figure 10 looks similar to the cell model of Figure 4.

\subsection{Asymmetric Photo Voltaic Module}

The asymmetric photovoltaic module consists of a physical series connection of cells (Figure 11). For each cell the corresponding shading can be adjusted by the module array parameter shadow. The division of the number of series connected cells by the number of bypass diodes, $\mathrm{ns} / \mathrm{nb}$, has to have zero remainder in order to model the bypass diodes symmetrically.

\subsection{Symmetric Plant}

The symmetric plant model is designed in the spirit of a symmetric module model as shown in Figure 10. For the plant model the number of series and parallel connected modules, nsModule and npModule are considered. The plant current $i$ and the plant voltage $v$ corre- 


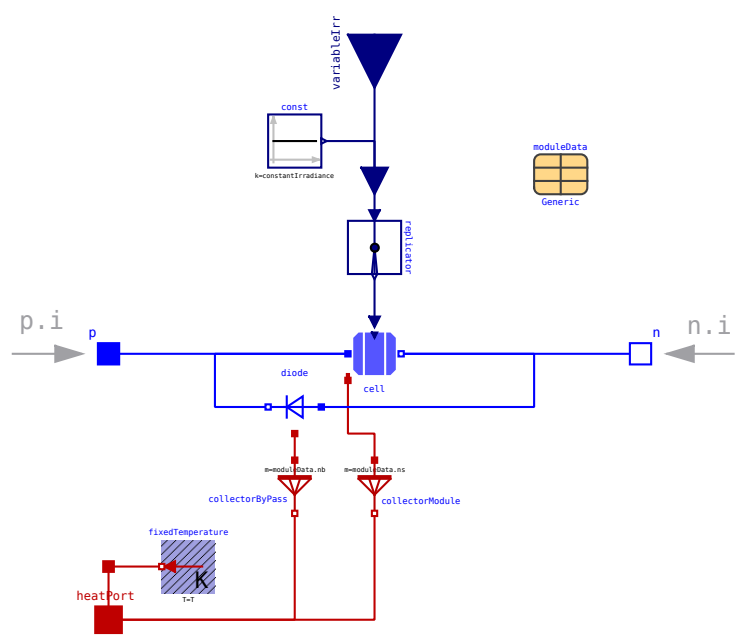

Figure 11. Modelica implementation of the module model SimpleModule

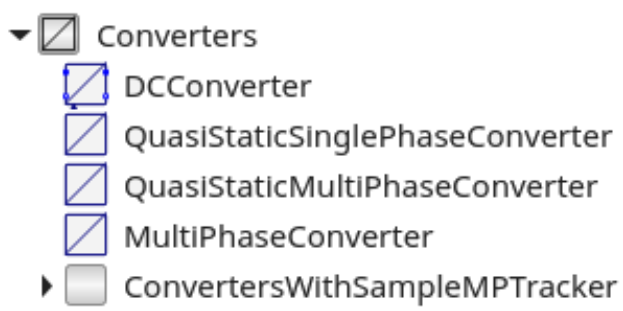

Figure 12. Converter models of the PhotoVoltaics Library

spond with module and cell currents and voltages by:

$$
\begin{aligned}
\mathrm{npPlant} * \mathrm{iModule} & =\mathrm{i} \\
\mathrm{iCell} & =\mathrm{iModule} \\
\mathrm{nsPlant} * \mathrm{vModule} & =\mathrm{v} \\
\mathrm{ns} * \mathrm{vCell} & =\text { vModule }
\end{aligned}
$$

\section{Converters}

The power conversion from a photovoltaic cell, module or plant to a DC, single or three phase AC grid is performed by means of converter models, see Figure 12. The converter models are all designed the same way considering the following characteristic behavior:

- Neither conduction nor switching losses are taken into account

- The converter models rely on ideal power conversion

- The voltage of the photovoltaic DC side can be adjusted (controlled) by a signal input

For the DC/DC conversion only, a converter model with integrated maximum power tracker is provided.

\section{Maximum Power Tracking}

In order to harvest the maximum energy, a photovoltaic plant has to be operated in the point of maximum power, see Figures 7 and 9. The photovoltaic DC side voltage has to be controlled such way that the maximum power point is reached. There are various maximum power tracking methods available in the literature. For the sake of simplicity there is only one discrete maximum power tracker implemented so far.

The implemented maximum power tracker is a block which evaluates the sensed power through a signal input. The output is the controlled photovoltaic DC voltage. The tracker samples the input power with a fixed sampling period. The output voltage is permanently changing in order to always follow changes of the maximum power point.

The initial setting is the voltage of the maximum power point according to the data sheet of the used modules. The output voltage gets increase by a voltage increment $\Delta v \cdot s$, where $s=-1$, so the output voltage actually gets decreased. If the sensed power is greater than the previously sensed power, the voltage will be decreased again. This procedure is performed until the actually sampled power gets smaller than the previously sampled power. In that case the sign $s$ will be altered. Then the output voltage starts increasing, again until the maximum power point is exceeded. This way, under steady state thermal and solar conditions, the output voltages is continuously changed between three different stages.

The experiment SimpleModuleMP investigates the maximum power tracking under varying irradiance conditions and a sampling time of one second. The initial irradiance is equal to $200 \mathrm{~W} / \mathrm{m}^{2}$. From 100 to $200 \mathrm{sec}$ onds the irradiance increases linearly up to $1000 \mathrm{~W} / \mathrm{m}^{2}$ and remains constant until 300 seconds. Figure 13 and 14 show the power and reference voltage versus time, respectively. The reference voltage starts with the voltage of the maximum power point $V_{\mathrm{mp} \text {,ref }}=24 \mathrm{~V}$, which refers to $1000 \mathrm{~W} / \mathrm{m}^{2}$. However, since the experiment starts with $200 \mathrm{~W} / \mathrm{m}^{2}$, the reference voltage is decreased to roughly $20 \mathrm{~V}$ in order to reach the actual maximum power point of approximately $30 \mathrm{~W}$ under these conditions. After increasing the irradiance to $1000 \mathrm{~W} / \mathrm{m}^{2}$ the reference voltage is controlled up to $24 \mathrm{~V}$ which is equal to the expected $V_{\mathrm{mp}, \text { ref }}=24 \mathrm{~V}$. From the voltage waveform the operating behavior of the controller can be observed. In quasi static operation the reference voltage is controlled upwards and downwards by $\Delta v$, having the maximum power point in between. The nonlinear increase of the reference voltage between 200 and 300 seconds is due to the fact that the irradiance and the maximum power of the module are not related linearly. Even though the power curve appears smooth in Figure 13, is also reveals discrete power changes when zooming into the curve. 


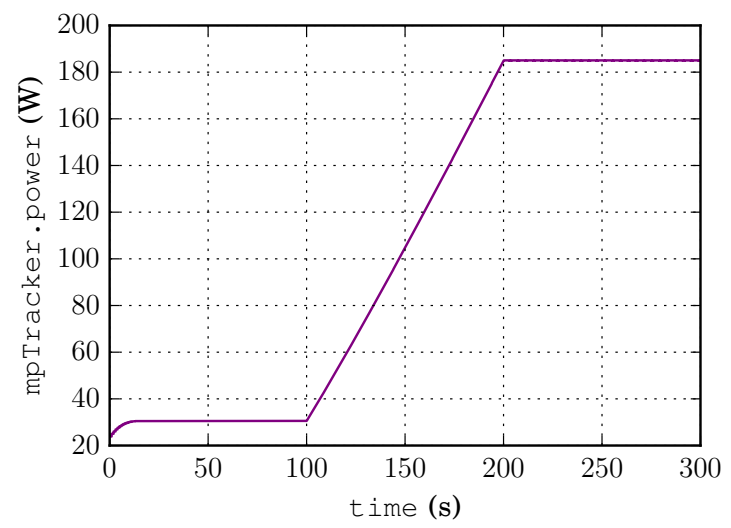

Figure 13. Example $\mathrm{S}$ impleModuleMP, power versus time

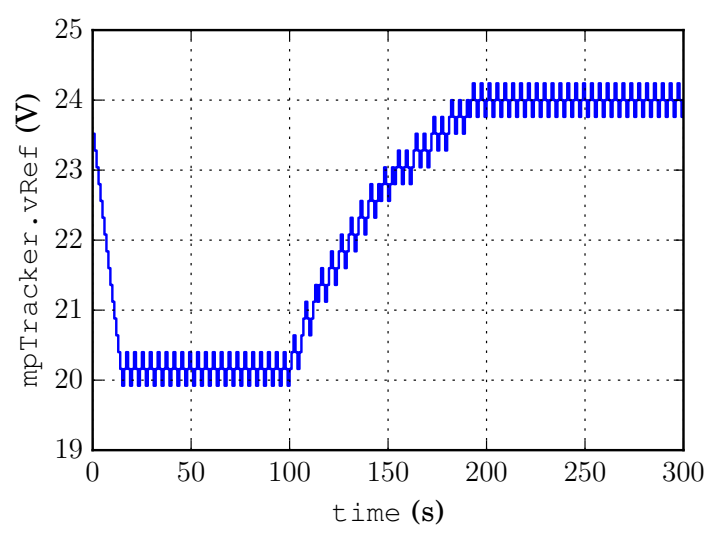

Figure 14. Example SimpleModuleMP, reference voltage versus time

\section{Irradiance Models}

In the PhotoVoltaics library terrestrial irradiance models are provided. The calculations are based on (Quaschning, 2011). The used equations are not discussed in this paper. However, the basic idea was to provide an analytic solar model that calculates the irradiance from the following parameters:

- Start day

- Start month

- Start year

- Time zone

- Longitude

- Latitude

- Reference irradiance (default value is $1000 \mathrm{~W} / \mathrm{m}^{2}$ )

- Angle of inclination, $\gamma$, of the photovoltaic module with respect to the horizontal plane

- Azimuth of the photovoltaic module orientation $(\mathrm{N}=$ $0^{\circ}, \mathrm{E}=90^{\circ}, \mathrm{S}=180^{\circ}, \mathrm{W}=270^{\circ}$, see Figure 21(c))

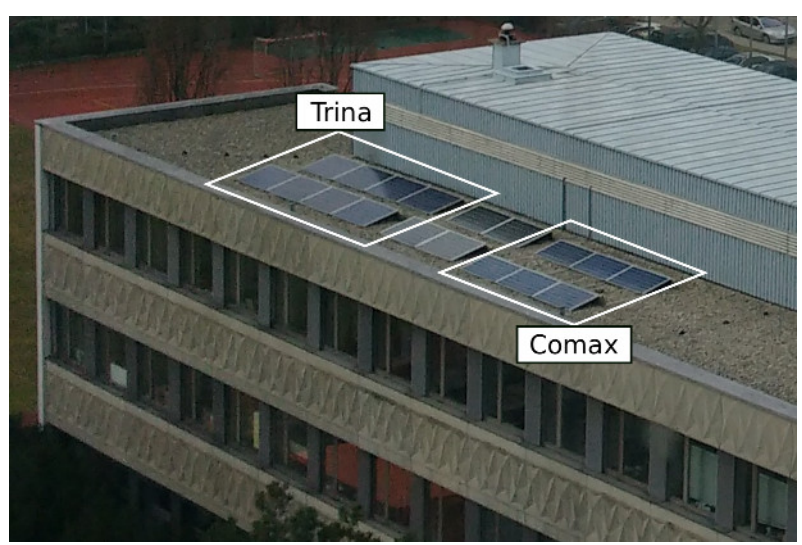

Figure 15. Photovoltaic plants of the TGM in Vienna

The start time of a simulation experiment refers to local time 00:00 of the indicated start day (and month and year). In this model, the following effects are not considered:

- Reflection

- Diffusion

- Visible obstacles in the vicinity of the photovoltaic plant

The purpose of the analytic solar model is to provide a basis for systemic investigations without having real weather conditions distorting the virtual experiments.

Since the Buildings library (Wetter, 2017) provides models to determine the effective irradiance based on statistical weather data, there are no extra models included in the PhotoVoltaics library to serve this purpose. Open access weather data of Vienna, Austria are included in the PhotoVoltaics library (EnergyPlus, 2017).

\section{Validation}

The PhotoVoltaics_TGM library is an additional library dedicated to the comparison of the simulation and measurement data of two photovoltaic plants located at the College of Engineering, TGM, in Vienna, Austria (Figure 15). The library relies on the PhotoVoltaics and the Buildings library (Wetter, 2017). The investigated plants are named after the manufacturer of the modules, Trina and Comax; see (Trina, 2017) and (Comax, 2017). The two plants are equipped with one irradiance sensor which allows the validation of the proposes cell and module models of the PhotoVoltaics library. For the two different plants one validation example, each, will be presented in this paper. The modules are aligned with the building, the direction is roughly south. The angle of inclination equals $10^{\circ}$.

For the validation of the PhotoVoltaics_TGM library a constant module temperature of $25^{\circ} \mathrm{C}$ is used. If necessary, measured or modeled temperature data could be fed to the thermal connector of the module. 


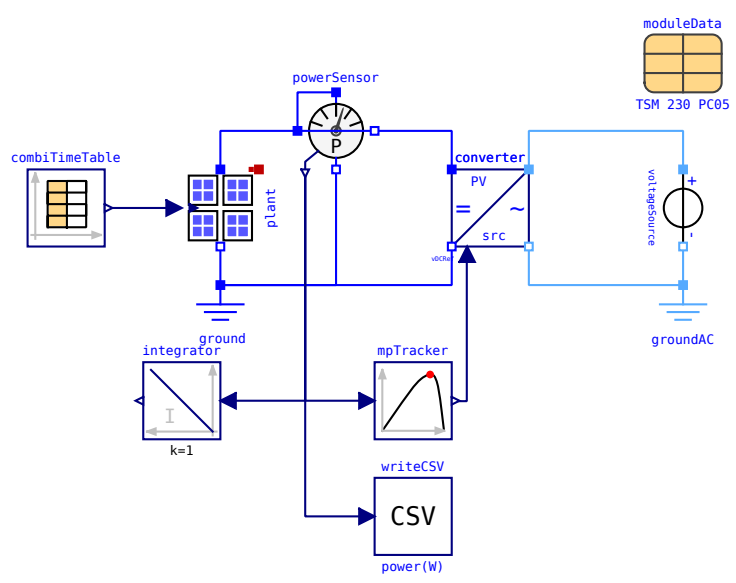

Figure 16. Model of the Trina plant of TGM in Vienna, reading irradiance data from a file

The first example, shown in Figure 16, refers to the Trina plant on 2016-06-29. The measured irradiance data are depicted in Figure 17 are fed to the irradiance input connector. A shadow of 0.1 is taken into account in the model, to consider the degradation due to pollution (Häberlin and Renken, 1999). The generated DC power is fed to the AC power grid by means a quasi static DC/AC converter including power tracking. The simulated power is also integrated to determine the energy harvest of this day. An additional interface block is used to write time versus power data directly to a CSV file to simplify the evaluation with a spreadsheet processing software.

The irradiance waveform of Figure 17 shows one significant drop at 16:30 caused by the shadow of the highriser school building, which is located next to the photovoltaic plants. The maximum irradiance reaches about $1000 \mathrm{~W} / \mathrm{m}^{2}$ and occurs at 13:30.

Since the power generated by the plant is directly proportional to the irradiance, the simulated DC power in Figure 18 shows the same drop at 16:30 as the irradiance. This figure also shows the measured DC and AC power of the converter. The measured DC power shows a high congruence with the simulated DC power. The measured $\mathrm{AC}$ power is smaller than the DC power due to the loss of the DC/AC converter. In the simulation, the converter loss is not taken into account. Consequently, there is no equivalent quantity in the simulation to be compared to the measured $\mathrm{AC}$ power.

The second example investigates the Comax plant at the TGM in Vienna on 2016-06.29. This plant has a smaller peak power than the Trina plant. The simulated DC power and the measured AC power are shown in Figure 19. Unfortunately, the converter of the Comax plant does not measure the DC power. Therefore, only the measured AC power can be compared to the simulated DC power. The difference between the two curves is again caused by the converter loss. However, by comparing the Figures 18 and 19 it can be roughly estimated that measurement and simulation again show a good agreement, if similar converter

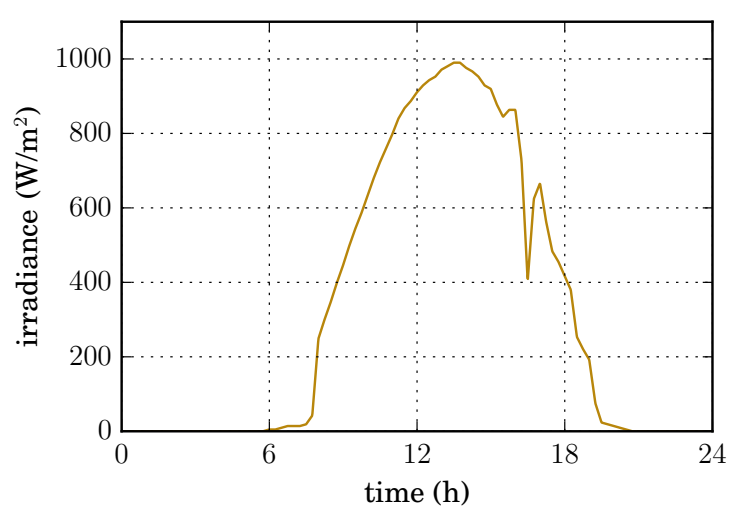

Figure 17. Measured irradiance at TGM in Vienna on 2016-0629

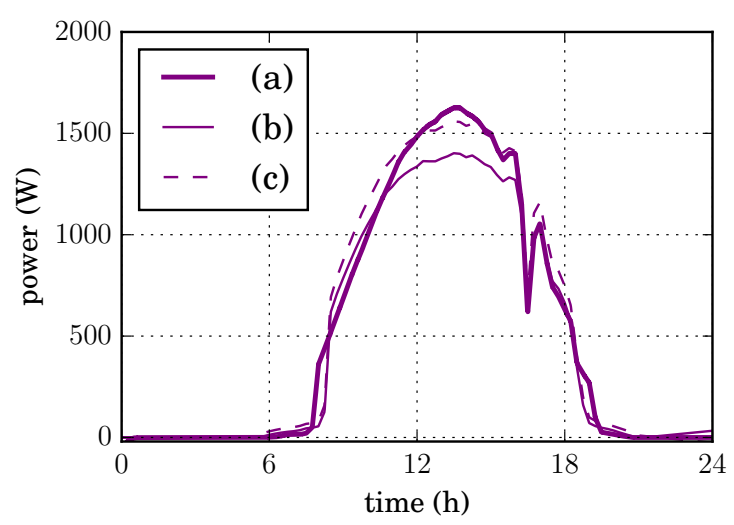

Figure 18. Power of the Trina plant at TGM in Vienna on 201606-29, (a) simulated DC power, (b) measured AC power and (c) measured DC power

losses for both cases are presumed.

The comparison of the measurement and simulation results of the two plants validates the presented PhotoVoltaics library.

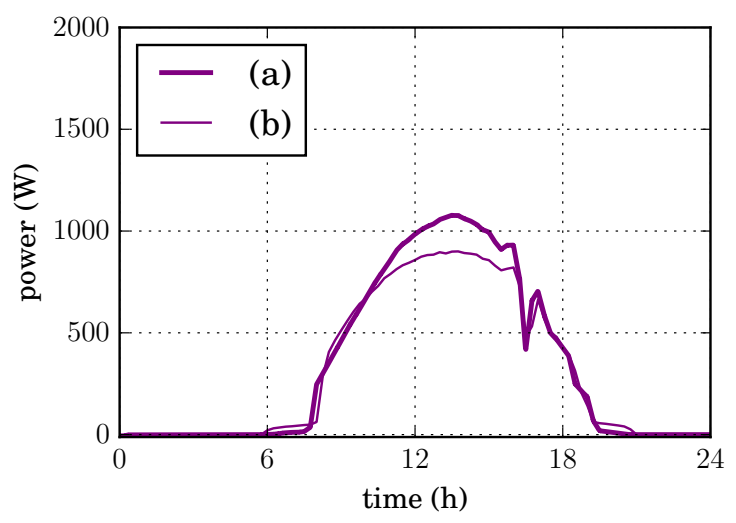

Figure 19. Power of the Comax plant at TGM in Vienna on 2016-06-29, (a) simulated DC power, (b) measured AC power and (c) measured DC power 


\section{Applications}

Typical applications of the PhotoVoltaics library are systemic investigations which include photovoltaics. Since all photovoltaic components are equipped with a thermal heat port, the influence of temperature on the operational behavior may be investigated. Particularly, the library is capable of investigating of the total energy consumption and generation of alternative building concepts including interaction with the power grid.

One special application of the PhotoVoltaics library is the Phileas rover of the Austrian Space Forum (Austrian Space Forum, 2017). This rover is equipped with four triangularly shaped solar panels. The four panels are equipped with photovoltaic cells and shape a pyramid in the upright position as shown in Figure 20(a). At the top of the pyramid all four panels are mechanically connected. This top point can be moved vertically only. The remaining two bottom points of each face can only move in the horizontal plane as sketched in Figure 20(b). So, by vertically adjusting the top point of the four panels, the total energy harvest of the panel configuration can be changed. The actual panel configuration is characterized by the inclination angle $\gamma$ of a panel as shown in Figure 20(a) and (b).

The Modelica model of the Phileas rover is depicted in Figure 21. If the position of one solar panel is known the position of other second panels is displaced by $90^{\circ}$, and so on. Therefore, the input connectors of the model are the inclination angle $\gamma$ and the azimuth of panel number 1. The inclination angles of the four panels are equal. The location of the sun is fixed in this model in order to allow systemic investigations. The irradiance of each of the four models is calculated and fed to the respective signal input of the panel. Each panel is connected with a DC/DC converter including maximum power tracker. The output sides of the four DC/DC converters are connected in parallel.

In the experiment SolarPyramidBatteryCharge a simplified investigation is made. The azimuths of the panels are kept constant and the inclination angle $\gamma$ is varied between 0 and $60^{\circ}$. The output connectors of the four parallel DC/DC converters are supplying a battery with constant voltage. The panel parameters are taken from a standard module. The actual geometric size of the four panels is not taken into account, since it cause a scaling of power only. An additional simplification of the experiment is done by using terrestrial solar irradiances instead of the irradiances on the Mars. The sun height is set to $22^{\circ}$ and the sun azimuth is equal to $260^{\circ}$ (see Figure 20(c)), i.e., the orientation of the sun is close to west. The four panels are oriented towards the main directions (panel $1=$ north, panel $2=$ east, panel $3=$ south, panel $4=$ west). The calculated powers of the four panels (1)-(4) and the sum power $(\Sigma)$ are depicted in Figure 22. When increasing the inclination angle $\gamma$ up to approximately $22^{\circ}$, the power of solar panel number 2 drops to zero. This is a conse-

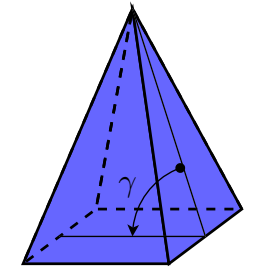

(a)

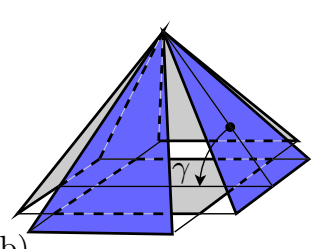

(b)

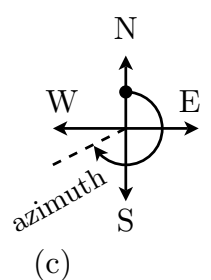

(c)
Figure 20. Solar panels of the Phileas rover (a) in most upright position and (b) in inclined position; (c) definition of the azimuth

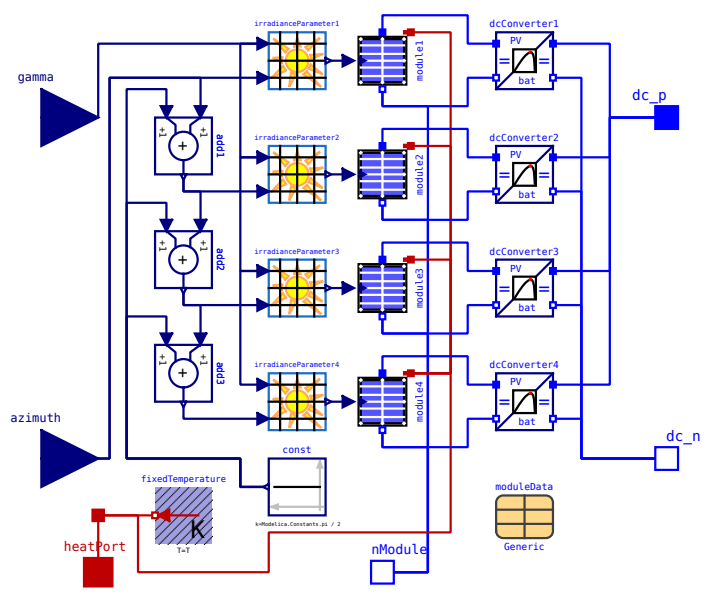

Figure 21. Model of the Phileas rover of the Austrian Space Forum (Austrian Space Forum, 2017)

quence of the inclination angle being greater than the sun height. The power of panel number 4 becomes the greatest, since it is oriented to west, close to the azimuth of the sun. All four panel powers start at the same starting power at $\gamma=0^{\circ}$, since they are then equally located in the plane. The total power shows a local maximum at $\gamma=0^{\circ}$ and a global maximum at $\gamma=42^{\circ}$.

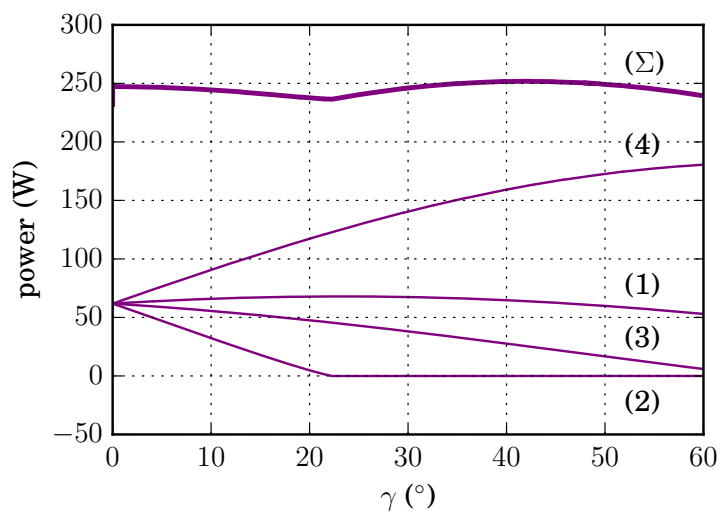

Figure 22. Power versus inclination angle $\gamma$ of panels (1)-(4) and $\operatorname{sum}(\Sigma)$ 


\section{Conclusions}

In this paper the open source PhotoVoltaics library was presented. Various photovoltaic components are explained based on typical data sheet parameters provided by manufacturers. Different models of cells, modules and plants are explained. Additional models of converters including maximum power tracking are described. In order to make systemic investigations, different analytic solar model are introduced.

Based on measurement data of two small photovoltaic plant at the TGM in Vienna, a validation of the library is performed. An application example of the Phileas rover of the Austrian Space Forum is investigated to demonstrate the potential of systemic investigations enabled by the PhotoVoltaics library.

\section{References}

OeWF Austrian Space Forum. The Phileas Rover, June 2017. URL http://oewf.org/en/polares-science/ phileas-rover/.

BuildSysPro. Edf's modelica library for buildings, districts and energy systems modelling, June 2017. URL https: //github.com/edf-enerbat/BuildSysPro.

Comax. Photo voltaic module data sheet Comax TSM 200 DC 01A, June 2017. URL http: //www.elektra.si/uploads/datoteke/trina_ tsm-195-200-205-210dc80.08_mono.pdf.

EnergyPlus. Weather data by location, europe wmo region 6 - austria, June 2017. URL https://energyplus. net/weather-location/europe_wmo_region_ 6/AUT//AUT_Vienna.Schwechat.110360_IWEC.

Heinrich Häberlin and Christian Renken. Allmähliche Reduktion des Energieertrags von Photovoltaikanlagen durch permanente Verschmutzung und Degradation. Bulletin SEVIVSE 10/99, 1999.

Christian Kral. Modelica PhotoVoltaics library, June 2017. URL https://github.com/christiankral/ PhotoVoltaics.

Yousef Mahmoud, W. Xiao, and H. H. Zeineldin. A simple approach to modeling and simulation of photovoltaic modules. IEEE Transactions on Sustainable Energy, 3(1):185186, January 2012.

PVSystems. Library toolbox for photovoltaic systems analysis, June 2017. URL https : / / git hub . com/mmanana / pvsystems.

Volker Quaschning. Regenerative Energiesysteme: Technologie - Berechnung - Simulation. Hanser, 7 edition, 2011.

C. Renken and H. Häberlin. Langzeitverhalten von netzgekoppelten Photovoltaikanlagen; Schlussbericht. Technical report, Berner Fachhochschule, Hochschule für Technik und Architektur (HTA) Burgdorf, 1999.

Trina. Photo voltaic module data sheet Trina TSM 230 PC05, June 2017. URL https://http: //www. franklin-electric.com.au/media/ 52578/Trina\%20TSM190-200DC01A_WW.pdf.

Michael Wetter. Modelica Buildings library, June 2017. URL https://github.com/lbl-srg/ modelica-buildings. 